\title{
PREFAZIONE ALL'EDIZIONE TEDESCA
}

Quando la Casa editrice osò prospettare l'idea di dare alle stampe una nuova edizione del trattato di Anatomia Patologica di Edoardo Kaufmann il rischio d'un tale piano sembrò scontato.

Ancora però più fortemente sentita dall'Autore principale, incaricato dalla Casa editrice, tu la difficoltà del compito di riassumere tutta l'esperienza raccolta sia pure in senso lato nel campo della patomorfologia nel corso degli ultimi decenni in una forma che lasciasse inalterato il carattere di un manuale e che il giovane o vecchio lettore, interessato allo studio delle basi anatomiche e della patogenesi delle malattie, potesse ritrovare in esso quel consigliere che per lui è sempre stato il "Kaufmann".

Non c'è particolare bisogno di sottolineare che un solo rielaboratore oggi non sarebbe stato piu in grado di ottemperare a questo compito.

La suddivisione della materia tra un numero di Autori, $i$ quali per il loro lavoro scientifico apparivano particolarmente versati in determinati campi, d'aver affidato questo compito, corrispose pienamente all'eredità che E. Kaufmann col suo libro ha lasciato nel mondo medico.

Da questo deriva naturalmente il pericolo che l'unità dell'opera possa andare perduta, e che singole parti non siano tra loro armonicamente accordate, come è possibile in un'opera di un solo $A$., e che perfino possano sorgere nelle parti dei singoli $A A$. contraddizioni nella concezione di particolari problemi.

Però d'altra parte ciò può in certi casi costituire per il lettore un vantaggio; attraverso tali contraddizioni la problematica di alcune teorie viene alla luce con più forza e non si ingenera cosi facilmente l'impressione che $i$ problemi debbano essere considerati come definitivamente risolti.

Il nuovo Kaujmann non sarà, come lo era il precedente, un trattato che espone con completezza tutta la letteratura.

Il suo compito consiste nell'esporre $i$ risultati più importanti dell'indagine anatomo-patologica in una forma che permetta anche al non patologo, $e$ specialmente a lui, una visione dei fondamenti morfologici delle malattie $e$ delle concezioni sulla loro patogenesi e che inoltre si addentri nei problemi importanti per il clinico ed il perito.

Si darà perciò valore al fatto che gli $A A$. in base alla propria esperienza, prendono posizione nei problemi discussi in letteratura e non rifuggono anche dalla critica. 
L'arte dell'esposizione, per non appesantire singole parti del testo, impone delle limitazioni, che sicuramente non sempre soddisferanno, ma che in ogni caso non sono fatte per nuocere alla completezza. Ciò vale specialmente per $i$ dati bibliografici.

Abbiamo deciso di non ripetere tutto l'elenco bibliografico della edizione precedente, ma dei lavori piu vecchi riportare generalmente solo quelli di valore storico o di importanza fondamentale, mentre $i$ nuovi lavori (con una necessaria selezione) vengono riportati nel maggior numero possibile.

Nella scelta delle figure furono ripetutamente presi $i$ magistrali disegni di E. Kaufmann su preparati macroscopici, altri sostituiti o completati con fotografie.

Maggior valore fu dato alle figure microscopiche, le quali nella loro grande prevalenza furono eseguite su microfotogrammi. Noi speriamo che il libro raggiungerà il suo scopo, di essere cioè per il lettore un consigliere ed una guida nel dedalo spesso inestricabile della letteratura e aiutarlo perciò, anche nei problemi della prevenzione delle malattie, della perizia e del trattamento, a prendere suggerimenti dal libro.

Alla Casa spetta il ringraziamento dell'A utore principale e dei collaboratori per aver coraggiosamente affrontato tutto il piano e per la sua fatica di venire incontro ai desideri degli $A A$. per far si che il libro anche esternamente nella presentazione rappresenti un'opera moderna.

\section{Martin StaemmLer}

Aquisgrana, primavera 1955 